\title{
Modelo de Markowitz aplicado a fondos de inversión en Bolivia
}

\author{
Markowitz model applied to investment funds in Bolivia
}

\section{Modelo de Markowitz aplicado a fundos de investimento na Bolívia}

\author{
Recibido: mayo 2019 \\ Arbitrado: junio 2019 \\ Publicado: septiembre 2019
}

\author{
《 Diego Coria Villca \\ coriabol@hotmail.com \\ ORCID 0000-0001-7982-7278
}

Universidad Andina Simón Bolívar, Bolivia

\section{RESUMEN}

En Bolivia se invierte en la bolsa de valores a través de las sociedades administradoras de fondos de inversión (SAFIs) quienes ofrecen 52 fondos de inversión, como en todo mercado financiero la decisión de dónde invertir es complicada. En este sentido, el estudio se propuso elaborar un portafolio óptimo de inversión en SAFIs que ofrezca el mayor rendimiento con un nivel de riesgo controlado. Con corte transversal, aplicando el modelo de Markowitz y la teoría de portafolios, se analizó el comportamiento diario de los instrumentos durante el periodo de julio-octubre de 2014. Como resultado se obtuvo el portafolio de mínima varianza con rendimiento de $1,87 \%$ y riesgo de $0,57 \%$ y el portafolio óptimo con rendimiento del $4,10 \%$ y riesgo del $3,65 \%$. Como conclusión se sabe que posible optimizar el rendimiento de inversión en SAFIs utilizando este modelo, asimismo, es factible aplicar el mismo tratamiento estadístico para otros periodos de tiempo.

Palabras clave: Fondos de inversión; portafolio de inversión; volatibilidad; riesgo; rendimiento

\section{RESUMO}

In Bolivia, investments are made in the stock market through "investment fund management companies" (SAFIs) who offer 52 investment funds, as in any financial market, the decision of where to invest is complicated. In this sense, the study set out to develop an optimal investment portfolio in SAFIs that offers the highest return with a controlled level of risk. With a cross-section, applying the Markowitz model and portfolio theory, the daily behavior of the instruments was analyzed during the period JulyOctober 2014. As a result, the portfolio with minimum variance was obtained with a yield of $1.87 \%$ and risk of $0.57 \%$ and the optimal portfolio with yield of $4.10 \%$ and risk of $3.65 \%$. As a conclusion, it is known that it is possible to optimize the return on investment in SAFIs using this model, also, it is feasible to apply the same statistical treatment for other time periods.

Key words: Investment funds; investment portfolio; volatility; risk; performance
Na Bolívia, os investimentos são feitos no mercado de ações por meio de "sociedades gestoras de fundos de investimento" (SAFIs) que oferecem 52 fundos de investimento, pois em qualquer mercado financeiro a decisão de onde investir é complicada. Nesse sentido, o estudo se propôs a desenvolver uma carteira ótima de investimentos em SAFIs que ofereça o maior retorno com um nível de risco controlado. Em cross-section, aplicando o modelo de Markowitz e a teoria da carteira, foi analisado o comportamento diário dos instrumentos durante o período de julho a outubro de 2014. Como resultado, obteve-se a carteira com variância mínima com rendimento de $1,87 \%$ e risco de $0,57 \%$ e a carteira ótima com rendimento de $4,10 \%$ e risco de $3,65 \%$. Em conclusão, sabe-se que é possível otimizar o retorno do investimento em SAFIs utilizando este modelo, da mesma forma, é possível aplicar o mesmo tratamento estatístico para outros períodos de tempo.

Palavras-chave: Fundos de inversão; Carteira de investimentos; Volatilidade; Risco; Desempenho 


\section{INTRODUCCIÓN}

$\mathrm{L}$ a bolsa de valores es un mercado organizado, en el que se realizan transacciones con títulos valores de oferta pública por medio de intermediarios autorizados, conocidos como casas de bolsa. Dependiendo del momento en que un título ingresa al mercado, estas negociaciones se transarían en el mercado primario o en el mercado secundario (Ross, 2009 \& Villarreal, 2008). Los recursos invertidos por medio de las bolsas de valores permiten tanto a las empresas como a los gobiernos, financiar proyectos productivos y de desarrollo que generan empleos y riqueza para un país. Los oferentes de estos recursos reciben a cambio la oportunidad de invertir en una canasta de instrumentos que les permite diversificar su riesgo optimizando sus rendimientos (Ross, 2009 \& Lawrence, 2012).

En Bolivia la bolsa de valores es un mercado complementario al Sistema Financiero Nacional, a su vez, las sociedades administradoras de fondos de inversión (SAFIs) tienen por objetivo administrar fondos de inversión, cuyos participantes son personas naturales o jurídicas que constituyen un fondo común de aportes de dinero en efectivo. Los accionistas son agencias de bolsa, bancos y compañías de seguros, su patrimonio está separado del patrimonio del fondo que administran (ASOBAN, 2013). Las SAFls establecen e implementan las políticas de inversión que persigue el fondo, realizando inversiones a favor de los participantes y a través del mercado de valores nacional y del extranjero, sus ingresos provienen de las comisiones que cobran a los aportantes por la administración del fondo (Castaño, 2008).

En el año 2013, el índice global de actividad económica de Bolivia tuvo un crecimiento de 6,5\%. A nivel sectorial, todas las actividades aportaron al crecimiento destacando entre los más relevantes hidrocarburos, transportes y almacenamiento, otras industrias, agropecuaria junto al continuo dinamismo de servicios financieros; estos cinco sectores explicaron más de dos terceras partes del crecimiento registrado a precios básicos. Este mismo año, el crédito del sistema financiero al sector privado presentó el crecimiento absoluto más alto en la historia económica del país y los depósitos continuaron aumentando a un ritmo importante. (BCB, 2014).

Este mercado de valores tiene participación predominante de la Bolsa Boliviana de Valores (BBV) y del Banco Central de Bolivia (BCB). La venta directa de valores al público se ha convertido en un mecanismo que favorece las condiciones de ahorro de la población, ofreciendo a las familias la seguridad de un título del $B C B$ y tasas de interés más altas que en las Entidades de Intermediación Financiera (EIF). Adicionalmente, estos títulos se constituyeron en un instrumento alternativo para regular la liquidez y señalizar las tasas pasivas del sistema financiero (BCB, 2014).

Frente a la liquidez presente en la sociedad boliviana, las SAFIs son una alternativa de inversión, captan aportes de personas naturales o jurídicas (participantes), constituyéndose en un importante canal del ahorro hacia la inversión. Asimismo, es una alternativa para aquellas personas que deseen participar con sus ahorros en el mercado de valores. Pero surgen algunas interrogantes ¿cuáles son los mejores fondos de inversión?, en este sentido, realizando revisiones bibliográficas 
se advierte que no existe indicador que muestre tal situación, entonces elegir entre la gran diversidad de fondos de inversión que ofertan las SAFIs se torna complicado, ¿será posible construir un portafolio de fondos de inversión?, ¿podrá aplicarse la teoría de portafolios de Markowitz en los fondos de inversión que administran las SAFIs?

Surge entonces el presente estudio que parte de la proposición teórica que afirma que es posible aplicar esta teoría a los fondos de inversión en Bolivia, el problema que ocupa el estudio es cómo seleccionar fondos de inversión para elaborar un portafolio óptimo que genere buenos rendimientos a menor riesgo. Así, el objetivo de la investigación fue elaborar un portafolio óptimo de fondos de inversión sustentado en la teoría de Markowitz que permita maximizar la rentabilidad a un riesgo determinado, con base en datos publicados por la ASFI periodo julio-octubre de 2014.

Este estudio se justifica por dos grandes contribuciones, la primera hace referencia al aporte práctico, ya que sirvió como asesoría científica para toda persona natural o jurídica con intenciones de invertir en valores, permitiéndoles conocer el portafolio óptimo de inversión, específicamente se dio alternativas de inversión a los participantes en las SAFIs de Bolivia en la gestión 2014. Con este antecedente, la investigación se justifica también porque probó científicamente que el modelo de Markowitz es aplicable al caso boliviano para determinar los mejores fondos de inversión con base en rendimiento, volatilidad, desempeño y correlación, por tanto, posible replicar el análisis para periodos actuales o futuros, constituyéndose en una herramienta clave para los inversionistas.

Se procede a presentar la base teórica y normativa en la que se enmarca el estudio, empezamos entonces explicando las características de los portafolios de inversión. Sabemos que un portafolio de inversión es un conjunto de activos seleccionados de un universo de alternativas existentes en los mercados financieros, el proceso de elección está sujeto a la diversificación, e implica la proporción de inversión que se destinará a cada activo financiero, esta acción permite equilibrar los rendimientos esperados y el riesgo que se obtienen de cada uno de los activos.

Lo atractivo de una inversión es el rendimiento que se puede obtener, pero la vulnerabilidad de los mercados frente a eventos económicos, sociales y políticos generan incertidumbre al inversionista, por lo tanto, toda inversión conlleva cierto nivel de riesgo. Sobre la base de revisiones bibliográficas, la teoría de portafolio de inversión se remonta al año de 1952 cuando apareció una publicación de Harry M. Markowitz "Portfolio Selection", en ella se muestra la composición de un portafolio utilizando conceptos básicos de estadística como la esperanza matemática y la desviación estándar y su relación con el rendimiento y el riesgo, de esta manera propone al inversionista que aborde el portafolio como un todo y no de manera particular por cada activo. En un portafolio existen diferentes alternativas de inversión que permita al inversionista seleccionar las más restables con un menor riesgo posible (Fabozzi; Markowitz; Gupta, 2008).

Miller y Modigliani (1958), indican cómo las políticas de dividendos y de financiamiento repercuten en el valor de la empresa y realizan grandes aportaciones a las finanzas corporativas. Posteriormente Sharpe (1964), con base en los resultados de Markowitz, desarrolló la teoría de 
equilibrio donde los precios de los activos se ajustarán para igualar la oferta y la demanda de cualquier activo riesgoso.

Habiendo ya presentado la base teórica sobre la cual descansa el estudio, se procede entonces a describir los fondos de inversión según la normativa vigente en Bolivia (ASFI, 2014):

El fondo de inversión es el patrimonio común autónomo y separado jurídica y contablemente de la sociedad administradora, constituido a partir de los aportes de personas naturales y jurídicas denominadas participantes, para su inversión en valores, bienes y demás activos determinados por la ley del mercado de valores y demás normativa aplicable, por cuenta y riesgo de los participantes. La propiedad de los participantes respecto al fondo de inversión se expresa a través de las cuotas de participación emitidas por el mismo. Las SAFIs establecen e implementan las políticas de inversión que persigue el fondo, realizando inversiones a discreción propia, a favor de los participantes del fondo y por cuenta y riesgo de estos. Las políticas de inversión son de conocimiento de los aportantes con anterioridad a su ingreso al fondo (ASFI, 2014).

La compra de cuotas es la operación en la que el participante mediante aportes de dinero adquiere cuotas de participación de un fondo de inversión, ya sea a través de la sociedad administradora en el caso de fondos de inversión abiertos o a través de intermediarios autorizados en mercado primario o secundario en el caso de fondos de inversión cerrados. Por su parte, la cuota de participación es una de las partes alícuotas, iguales y de similares características en las que se divide el patrimonio de un fondo de inversión y que expresa los aportes de los participantes. Asimismo, la venta de cuotas es la operación mediante la cual el participante hace líquidas o convierte en dinero las cuotas de un fondo de inversión cerrado a través del mercado secundario. Finalmente, la entidad calificadora de riesgos es una sociedad anónima de objeto exclusivo autorizada por ASFI para calificar riesgos en el mercado de valores, conforme a lo previsto por la ley del mercado de valores y el reglamento para entidades calificadoras de riesgo. Asimismo, se encuentran comprendidas dentro de esta definición las entidades calificadoras de riesgo constituidas en el extranjero que se encuentren autorizadas para prestar el servicio de calificación de riesgo en el país conforme a lo previsto por la normativa pertinente (ASOBAN, 2014).

Según normativa, los fondos de inversión se clasifican según tipo (abiertos y cerrados), plazo, rentabilidad, políticas de inversión, moneda, cuotas de participación y características propias del fondo (ASFI, 2015).

Fondos de inversión abierto. El fondo es del tipo abierto dadas sus características de variabilidad respecto a su patrimonio, los instrumentos en los que se invierte son de renta fija o variable y las cuotas de participación colocadas entre el público son redimibles directamente por el fondo siendo su plazo de duración indefinido. El fondo se clasifica de renta fija dadas las características de los valores en los cuales invierte. El fondo se denomina Fondo de Inversión Abierto de Mediano plazo o Corto Plazo, debido a que la duración promedio ponderada de la cartera deberá ser menor a 360 o 1080 días. 
Fondos de inversión cerrado. El fondo es del tipo cerrado cuyo patrimonio es fijo y las cuotas de participación colocadas entre el público no son redimibles directamente por el fondo, salvo en las circunstancias y procedimientos dispuestos específicamente por su reglamento interno como liquidación por vencimiento del plazo del fondo y el procedimiento de transferencia, fusión, liquidación y disolución. Las cuotas de participación se encuentran autorizadas e inscritas en el registro de mercado de valores de la autoridad de supervisión del sistema financiero, así como la oferta pública de las mismas y pueden ser negociadas en la BBV. La colocación primaria de las cuotas de participación se realizará en la BBV y podrán posteriormente ser negociadas de forma bursátil y extrabursátil en el mercado secundario.

\section{MÉTODO}

$\mathrm{S}$ e trata de una investigación descriptiva que toma como unidades observables a los fondos de inversión administrados por las SAFIs, analizando el comportamiento diario de los instrumentos durante el periodo de julio-octubre de 2014, siendo un estudio transversal. Con relación a los métodos empíricos, se aplicó la observación para recopilar información referente a rendimientos diarios de los fondos de inversión. Asimismo, se empleó el modelo de optimización de portafolios de Markowitz para construir los portafolios eficientes, de mínima varianza y el óptimo (Markowitz, 1959 \& Ross, 2009).

Los pasos que se siguieron fueron cinco: 1) Se obtuvo información histórica de los fondos de inversión de Bolivia a través de la ASFI, periodo julio-octubre de 2014. 2) Se exploró los rendimientos esperados, desviación estándar, asimetría, curtosis, varianza, el coeficiente de variación y el análisis de correlación lineal de Pearson. 3) Se segmentó los fondos según tipo (abierto y cerrado) y moneda (moneda nacional, dólares americanos y unidad de fomento a la vivienda UFVs). 4) Se aplicó la teoría de Markowitz en el modelo de portafolio para los fondos de inversión. 5) Se presentaron resultados acompañados de la interpretación correspondiente.

Los postulados aplicados para aplicar la teoría de Markowitz en el modelo de portafolio de Bolivia se enuncian a continuación (Markowitz, 1952; Ross, 2009).

Cálculo de la distribución normal de rendimientos $\mu$ y $\sigma$ :

$$
\begin{gathered}
\mu=E(R)=\int_{-\infty}^{\infty} \frac{1}{\sigma \sqrt{2 \pi}} e^{-\frac{1}{2}\left(\frac{R-\mu}{\sigma}\right)^{2}} d R \\
\operatorname{var}(R)=\sigma^{2}=E[R-E(R)]=E\left[R^{2}\right]-[E(X)]^{2}
\end{gathered}
$$

El rendimiento del portafolio es un promedio ponderado de los rendimientos esperados $E\left(R_{1}\right), E\left(R_{2}\right), \ldots, E\left(R_{n}\right)$ de un grupo de activos diversificados. 


$$
R_{p}=\sum_{i=1}^{n} w_{i} E\left(R_{i}\right)
$$

Donde:

$E\left(R_{i}\right)=$ Rendimiento esperado del activo $i$

$w_{i}=$ proporción invertida en el activo $i$

$\sum_{i=1}^{n} w_{i}=1$

$n=$ total de activos que se analizan.

El riesgo del portafolio,

$$
\sigma_{p}=\sqrt{\sum_{i=1}^{n} \sum_{j=1}^{n} w_{i} w_{j} \sigma_{i j}}
$$

Aplicando la teoría de portafolios la varianza del portafolio se calculó:

$$
\sigma_{P}^{2}=w^{2} \sigma_{X}^{2}+(1-w)^{2} \sigma_{Y}^{2}+2 w(1-w) \rho_{X Y} \sigma_{X} \sigma_{Y}
$$

Para $\rho_{X Y}=1$, la varianza del portafolio se calculó:

$$
\sigma_{P}^{2}=w^{2} \sigma_{X}^{2}+(1-w)^{2} \sigma_{Y}^{2}+2 w(1-w) \sigma_{X} \sigma_{Y}=\left[w \sigma_{X}+(1-w) \sigma_{Y}\right]^{2}
$$

y el riesgo del portafolio será:

$$
\sigma_{P}=w \sigma_{X}+(1-w) \sigma_{Y}
$$

La proporción $w$ con la cual se obtiene el riesgo mínimo cuando $\rho_{X Y}=1$ es,

$$
w=\frac{\sigma_{Y}^{2}-\sigma_{X} \sigma_{Y}}{\sigma_{X}^{2}+\sigma_{Y}^{2}-2 \sigma_{X} \sigma_{Y}}=\frac{\sigma_{Y}\left(\sigma_{Y}-\sigma_{X}\right)}{\left(\sigma_{Y}-\sigma_{X}\right)^{2}}=\frac{\sigma_{Y}}{\sigma_{Y}-\sigma_{X}}
$$

Para $\rho_{X Y}=-1$, de manera análoga al anterior caso se procede:

$$
\sigma_{P}^{2}=w^{2} \sigma_{X}^{2}+(1-w)^{2} \sigma_{Y}^{2}+2 w(1-w)(-1) \sigma_{X} \sigma_{Y}=\left[w \sigma_{X}-(1-w) \sigma_{Y}\right]^{2}
$$

y el riesgo del portafolio será.

$$
\sigma_{P}= \pm\left[w \sigma_{X}-(1-w) \sigma_{Y}\right]
$$

La proporción $w$ con la cual se obtiene el riesgo cero. 


$$
w=\frac{\sigma_{Y}^{2}+\sigma_{X} \sigma_{Y}}{\sigma_{X}^{2}+\sigma_{Y}^{2}+2 \sigma_{X} \sigma_{Y}}=\frac{\sigma_{Y}\left(\sigma_{Y}+\sigma_{X}\right)}{\left(\sigma_{X}+\sigma_{Y}\right)^{2}}=\frac{\sigma_{Y}}{\sigma_{X}+\sigma_{Y}}
$$

Para $\rho_{X Y}=0$, la varianza del portafolio es,

$$
\sigma_{P}^{2}=w^{2} \sigma_{X}^{2}+(1-w)^{2} \sigma_{Y}^{2}
$$

Riesgo del portafolio:

$$
\sigma_{P}=\sqrt{w^{2} \sigma_{X}^{2}+(1-w)^{2} \sigma_{Y}^{2}}
$$

Para determinar el portafolio óptimo se utilizó el concepto de desempeño del portafolio, aplicando el índice de Sharpe, que mide el exceso de rentabilidad sobre la tasa de interés libre de riesgo.

$$
I S_{p}=\frac{R_{p}-R_{f}}{\sigma_{p}}
$$

Donde:

$$
\begin{aligned}
& R_{p}=\text { Rendimiento del portafolio eficiente } \\
& R_{f}=\text { Tasa libre de riesgo } \\
& \sigma_{p}=\text { Riesgo del portafolio eficiente }
\end{aligned}
$$

\section{RESULTADOS}

$\mathrm{E}$ $\mathrm{n}$ el mercado de valores de Bolivia operan las siguientes 10 SAFIs: a) BISA SAFI S.A. administra 6 fondos de inversión abiertos y cerrados, en bolivianos y dólares, con calificación AA2 y A2. b) BNB SAFI S.A. administra 6 fondos de inversión abiertos y cerrados, en bolivianos y dólares, con calificación AAA, AA2, AA1 y A1. c) Capital+SAFI S.A. administra 2 fondos de inversión, ambos cerrados y en bolivianos, con calificación A1 y A2. d) Credifondo SAFI S.A. administra 4 fondos de inversión abiertos, en bolivianos y dólares, con calificación AA2 y A3. e) Fortaleza SAFI S.A. administra 15 fondos de inversión abiertos y cerrados, en bolivianos, dólares y UFVs, con calificación AAA, AA1, AA2, AA3, A3 y BB1. f) Marca Verde SAFI S.A. administra 2 fondos de inversión cerrados, en bolivianos y dólares, con calificación AA3. g) Mercantil Santa Cruz SAFI A.A. administra 6 fondos de inversión abiertos y cerrados, en bolivianos y dólares, con calificación AA2, AA1 y A2. h) Panamerican SAFI S.A. administra 2 fondos de inversiones cerradas y en bolivianos, con calificación A2 y A3. i) SAFI Unión S.A. administra 5 fondos de inversión abiertos y cerrados, en bolivianos y dólares, con calificación AA1, AA2 y AA3. j) Santa Cruz Investment SAFI S.A. administra 4 fondos de inversión abiertos y cerrados, en bolivianos y dólares, con calificación AA3 y A1. 
Del total de fondos administrados por las SAFIs en Bolivia, en la tabla 1 se presenta un resumen, clasificando según tipo, riesgo y moneda.

Tabla 1. Matriz de correlación del portafolio.

\begin{tabular}{|c|c|c|c|c|c|c|c|c|c|c|c|c|c|c|}
\hline \multirow{2}{*}{\multicolumn{2}{|c|}{ TIPO DE FONDO }} & \multicolumn{8}{|c|}{ CALIFICACIÓN DE RIESGO } & \multirow{2}{*}{ Total } & \multicolumn{3}{|c|}{ MONEDA } & \multirow{2}{*}{ Total } \\
\hline & & A1 & A2 & A3 & AA1 & AA2 & AA3 & AAA & BB1 & & BOB & UFV & USD & \\
\hline \multirow{2}{*}{ Abierto } & Recuento & 1 & 0 & 1 & 4 & 18 & 4 & 2 & 0 & 30 & 13 & 1 & 16 & 30 \\
\hline & $\%$ del total & $1,9 \%$ &, $0 \%$ & $1,9 \%$ & $7,7 \%$ & $34,6 \%$ & $7,7 \%$ & $3,8 \%$ &, $0 \%$ & $57,7 \%$ & $25,0 \%$ & $1,9 \%$ & $30,8 \%$ & $57,7 \%$ \\
\hline \multirow{4}{*}{ Cerrado } & Recuento & 3 & 4 & 2 & 3 & 2 & 6 & 0 & 2 & 22 & 17 & 0 & 5 & 22 \\
\hline & $\%$ del total & $5,8 \%$ & $7,7 \%$ & $3,8 \%$ & $5,8 \%$ & $3,8 \%$ & $11,5 \%$ &, $0 \%$ & $3,8 \%$ & $42,3 \%$ & $32,7 \%$ &, $0 \%$ & $9,6 \%$ & $42,3 \%$ \\
\hline & Total & 4 & 4 & 3 & 7 & 20 & 10 & 2 & 2 & 52 & 30 & 1 & 21 & 52 \\
\hline & $\%$ del total & $7,7 \%$ & $7,7 \%$ & $5,8 \%$ & $13,5 \%$ & $38,5 \%$ & $19,2 \%$ & $3,8 \%$ & $3,8 \%$ & $100,0 \%$ & $57,7 \%$ & $1,9 \%$ & $40,4 \%$ & $100,0 \%$ \\
\hline
\end{tabular}

Según la clasificación (véase tabla), es de resaltar que existe mayor participación de fondos de inversión abiertos (57,7\%), asimismo el 32,7\% opera en fondos cerrados en moneda nacional. Los fondos de inversión están calificados entre BB1- AAA, implica que cuentan con capacidad de pago hasta muy alta. El 34,6\% de los fondos tienen una calificación de riesgo de AA2 y son abiertos (alta capacidad de pago y dentro de ella el nivel medio). El 11,5\% de los fondos cerrados tienen una calificación de riesgo AA3.

Se presentan los resultados del estudio de los fondos de inversión según diferentes clasificaciones, el periodo de análisis se determinó desde el primero de julio hasta 22 de octubre de 2014, contabilizando 114 días.

\section{Fondos de inversión de tipo abierto}

Los fondos de inversión del tipo abierto que operan en el mercado de valores son 30, de los cuales 13 operan en moneda nacional, 1 en UFV y 16 en moneda extranjera. Para el análisis se excluye el fondo en moneda UFV por generar pérdidas durante el periodo.

Se procedió a la exploración de los fondos de inversión abierto, utilizando conceptos propios de estadística como valor esperado, varianza, desviación típica (volatilidad), coeficiente de variación, la mediana, el coeficiente de desempeño y asumiendo que los rendimientos siguen una distribución normal. Se observó alta volatilidad, a mayor rendimiento la volatilidad varia, el mejor fondo de inversión abierto en bolivianos es de la serie SFM, por presentar la menor volatilidad con relación a los fondos del mismo tipo, siendo su rendimiento esperado de 2,355\% y corresponde a Mercantil Santa Cruz SAFI S.A.

En relación a los resultados para los fondos de inversión abiertos en dólares americanos, se observa alta volatilidad con rendimientos entre $-200 \%$ hasta $200 \%$, el fondo con menor volatilidad es RAC, e implica mayor desempeño del grupo, por lo tanto es elegido como mejor fondo de 
inversión abierto en moneda extranjera. Además, los mejores fondos seleccionados del grupo que presentan menor volatilidad y mayor rendimiento son: HOR BSK, CFO, CCP y FFI.

\section{Fondos de inversión del tipo cerrado}

Los fondos cerrados en moneda nacional presentan volatilidades muy altas, reflejando rendimientos negativos mayores que $200 \%$. El fondo de inversión cerrado en moneda nacional con menor volatilidad y mayor desempeño es MFC. Los mejores fondos se seleccionan a partir del concepto de menor volatilidad y están alrededor del mejor fondo con relación al rendimiento total esperado, entre ellos se tiene: RAP, FPP_B, IFI, FAE, PUC, SMC, MIC_B, MIC_D y FFY.

Para el caso de los fondos cerrados en dólares, de la misma manera los fondos presentan muy alta volatilidad, reflejando pérdidas hasta el $800 \%$. El fondo con menor volatilidad y mayor desempeño es GAC, siendo el mejor fondo cerrado en dólares y los demás carecen de confiabilidad.

\section{Correlación de los fondos de inversión}

De los 52 fondos de inversión vigentes en Bolivia, tanto abiertos y cerrados en moneda nacional y extranjera, se seleccionaron 22 mejores fondos de inversión en Bolivia y son: OFI, SFM, OPU, FOI, CMR, RAC, HOR, BSK, CFO, CCP, FFI, MFC, RAP, FPP_B, IFI, FAE, PUC, SMC, MIC_B, MIC_D, FFY y GAC. Realizando un análisis de correlación a los 22 fondos, por medio del análisis de correlación lineal de Pearson, se determinó que 9 componen un portafolio que no presenta correlación positiva y cuyos fondos están incorrelacionados: MIC_D, MFC, SMC, CMR, RAC, GAC, $\mathrm{FFI}$, FOI y CFO.

En consecuencia, el portafolio está compuesto por 9 fondos de inversión (MIC_D, MFC, SMC, CMR, RAC, GAC, FFI, FOI, CFO) que no presentan correlación positiva (tabla 2).

Tabla 2. Matriz de correlación del portafolio eficiente.

\begin{tabular}{lrrrrrrrrr}
\hline FONDO & RAC & MFC & GAC & FFI & FOI & CFO & SMC & MIC_D & CMR \\
\hline RAC & 1 & $-0,003$ & 0,024 & $-0,008$ & $-0,014$ & $-0,067$ & $-0,001$ & 0,006 & $-0,03$ \\
Sig. & & 0,486 & 0,402 & 0,468 & 0,439 & 0,239 & 0,495 & 0,474 & 0,376 \\
MFC & $-0,003$ & 1 & 0,013 & 0,005 & 0,022 &,$- 161^{*}$ & $-0,065$ & 0,103 & 0,127 \\
Sig. & 0,486 & & 0,446 & 0,477 & 0,407 & 0,043 & 0,245 & 0,138 & 0,089 \\
GAC & 0,024 & 0,013 & 1 & $-0,034$ & $-0,02$ & $-0,076$ & $-0,141$ & 0,108 & $-0,076$ \\
Sig. & 0,402 & 0,446 & & 0,361 & 0,415 & 0,212 & 0,067 & 0,127 & 0,211 \\
FFI & $-0,008$ & 0,005 & $-0,034$ & 1 & $-0,036$ & $-0,066$ & 0,03 & $-0,036$ & 0,062 \\
Sig. & 0,468 & 0,477 & 0,361 & & 0,353 & 0,244 & 0,376 & 0,352 & 0,257 \\
FOI & $-0,014$ & 0,022 & $-0,02$ & $-0,036$ & 1 & 0,022 & $-0,053$ & 0,083 & $-0,141$ \\
Sig. & 0,439 & 0,407 & 0,415 & 0,353 & & 0,407 & 0,289 & 0,189 & 0,067 \\
\hline
\end{tabular}




\begin{tabular}{lrrrrrrrrr}
\hline FONDO & RAC & MFC & GAC & FFI & FOI & CFO & SMC & MIC_D & CMR \\
\hline CFO & $-0,067$ &,$- 161^{*}$ & $-0,076$ & $-0,066$ & 0,022 & 1 & $-0,038$ & 0,116 & 0,095 \\
Sig. & 0,239 & 0,043 & 0,212 & 0,244 & 0,407 & & 0,342 & 0,11 & 0,157 \\
SMC & $-0,001$ & $-0,065$ & $-0,141$ & 0,03 & $-0,053$ & $-0,038$ & 1 &,$- 201^{*}$ & 0,058 \\
Sig. & 0,495 & 0,245 & 0,067 & 0,376 & 0,289 & 0,342 & & 0,016 & 0,269 \\
MIC_D & 0,006 & 0,103 & 0,108 & $-0,036$ & 0,083 & 0,116 &,$- 201^{*}$ & 1 & 0,003 \\
Sig. & 0,474 & 0,138 & 0,127 & 0,352 & 0,189 & 0,11 & 0,016 & & 0,486 \\
CMR & $-0,03$ & 0,127 & $-0,076$ & 0,062 & $-0,141$ & 0,095 & 0,058 & 0,003 & 1 \\
\hline Sig. & $\mathbf{0 , 3 7 6}$ & $\mathbf{0 , 0 8 9}$ & $\mathbf{0 , 2 1 1}$ & $\mathbf{0 , 2 5 7}$ & $\mathbf{0 , 0 6 7}$ & $\mathbf{0 , 1 5 7}$ & $\mathbf{0 , 2 6 9}$ & $\mathbf{0 , 4 8 6}$ & \\
\hline
\end{tabular}

*La correlación es significativa al nivel 0,05 (unilateral).

Como se puede ver en la tabla 2, la matriz de correlación del portafolio no contiene correlación entre los fondos. Asimismo, se observó que en el caso de los fondos MIC_B y MIC_D existe una perfecta correlación y significativa, en tal sentido MIC_B no ingresa al portafolio (se discrimina). En la tabla 3 se muestra la clasificación de cada uno de los fondos seleccionados.

Tabla 3. Fondos que conforman el portafolio eficiente.

\begin{tabular}{|c|c|c|c|c|}
\hline Serie & Fondos de Inversión & $\begin{array}{l}\text { Cal. de } \\
\text { Riesgo }\end{array}$ & Tipo & Moneda \\
\hline \multicolumn{5}{|c|}{ BISA SAFI S.A. } \\
\hline GAC & Gestión Activa Fondos de Inversión Cerrado & AA2 & Cerrado & USD \\
\hline MFC & $\begin{array}{l}\text { Fondo de Microfinancieras Fondo de Inversión Cerrado } \\
\text { (MICROFIC) }\end{array}$ & $\mathrm{A} 2$ & Cerrado & $\mathrm{BOB}$ \\
\hline \multicolumn{5}{|c|}{ Capital + SAFI S.A. } \\
\hline SMC & Sembrar Micro Capital Fondo de Inversión Cerrado & $\mathrm{A} 2$ & Cerrado & BOB \\
\hline \multicolumn{5}{|c|}{ Credifondo SAFI S.A. } \\
\hline CFO & $\begin{array}{l}\text { Credifondo Renta Fija, Fondo de Inversión Abierto a } \\
\text { Mediano Plazo }\end{array}$ & AA2 & Abierto & USD \\
\hline CMR & $\begin{array}{l}\text { Credifondo + Rendimiento Fondo de Inversión Abierto a } \\
\text { Mediano Plazo }\end{array}$ & A3 & Abierto & $\mathrm{BOB}$ \\
\hline \multicolumn{5}{|c|}{ Fortaleza SAFI S.A. } \\
\hline FOI & $\begin{array}{l}\text { Fortaleza Interés + Fondo de Inversión Abierto Corto } \\
\text { Plazo }\end{array}$ & AA2 & Abierto & $\mathrm{BOB}$ \\
\hline $\mathrm{FFI}$ & $\begin{array}{l}\text { Fondo de Inversión Cerrado Fortaleza Factoring } \\
\text { Internacional }\end{array}$ & AA3 & Cerrado & USD \\
\hline MIC_D & Microfinanzas - Fondo de Inversión Cerrado Serie D & AA1 & Cerrado & $\mathrm{BOB}$ \\
\hline \multicolumn{5}{|c|}{ Santa Cruz Investment SAFI S.A. } \\
\hline RAC & Renta Activa Fondo de Inversión Abierto Corto Plazo & AA3 & Abierto & USD \\
\hline
\end{tabular}

Estos 9 fondos de inversión son los que pueden generar mejor portafolio de inversión para la presente investigación, de los cuales 5 fondos son cerrados y 4 abiertos, calificación de los fondos entre buena y alta capacidad de pago A3-AA2 (ver tabla 3). 


\section{Construcción de los portafolios eficientes y mínima varianza}

Se presentas las gráficas de series de tiempo para los 9 fondos de inversión seleccionados (gráfico 1).
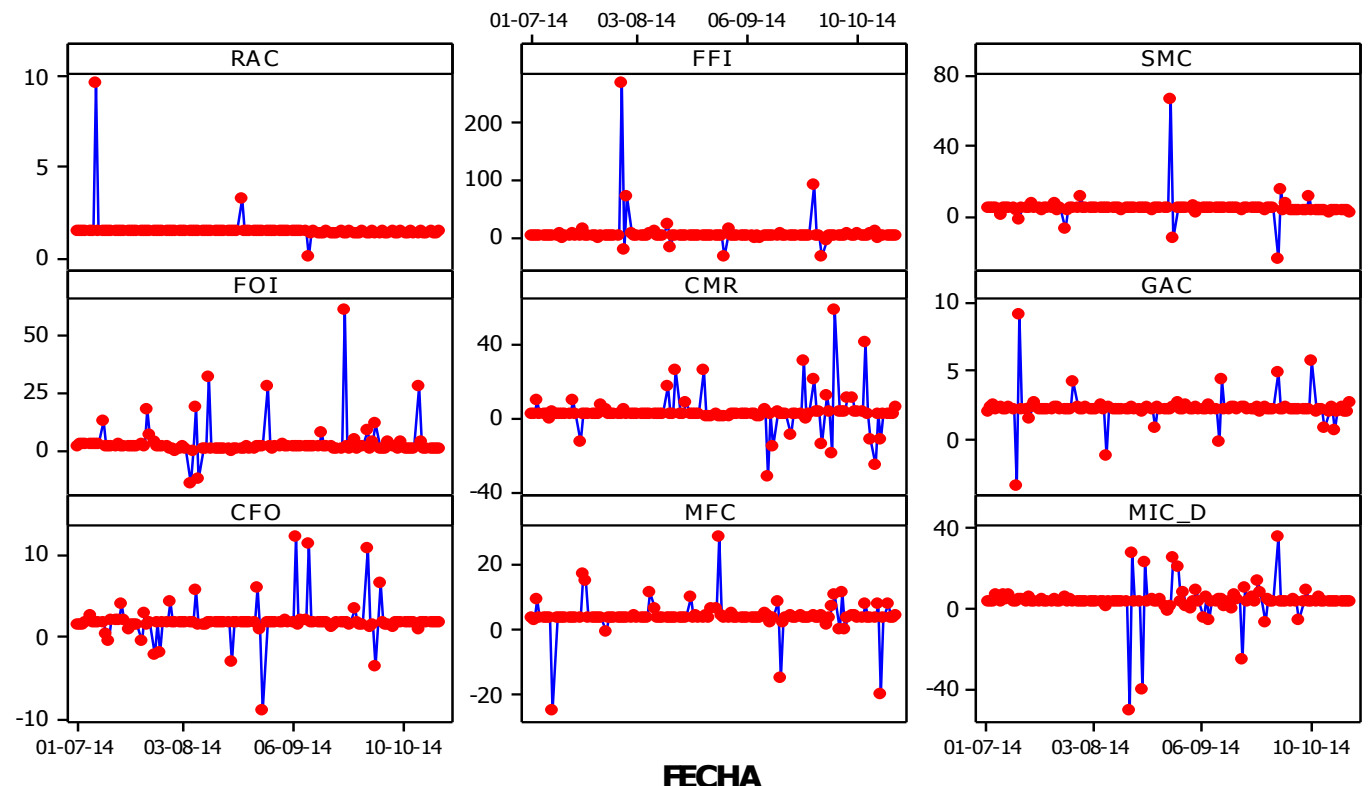

FECHA

Gráfico 1. Series de tiempo de 9 fondos de inversión, portafolio eficiente.

Analizado el gráfico 1, se afirma que el orden de los fondos con relación a la asimetría es RAC, FFI, SMC, FOI, CMR, GAC, CFO, MFC y MIC_D. Un inversionista adverso al riesgo toma la decisión en invertir en los fondos RAC, FFI, SMC, FOI, CMR y GAC. A su vez, la mayor concentración de los fondos con relación al rendimiento y volatilidad se encuentra localizada entre $(3,06: 7,89)$ y $(4,22: 5,19)$,

Según se observa en el gráfico 2, es posible que el portafolio óptimo esté entre el rango mencionado.

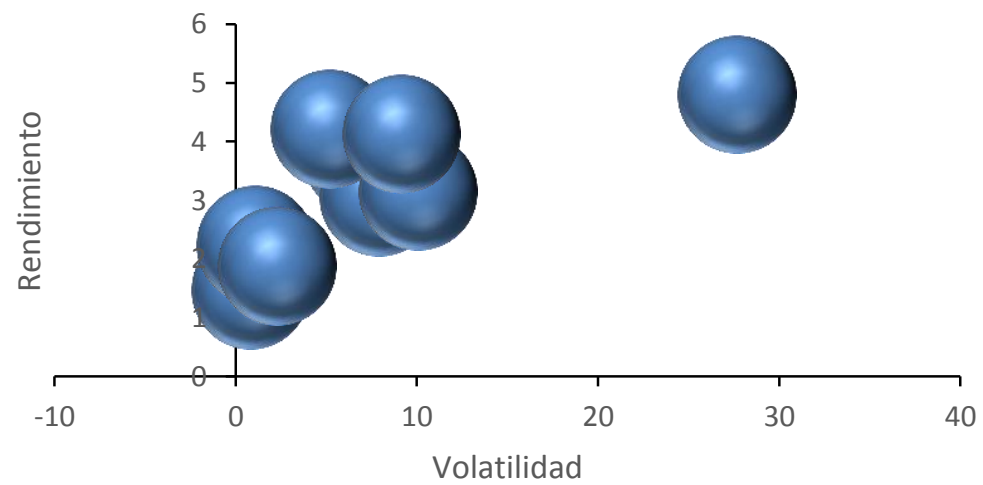

Gráfico 2. Rendimiento y volatilidad de 9 fondos. 
A continuación, en la tabla 4 se muestra la matriz de varianzas-covarianzas del portafolio.

Tabla 4. Matriz de varianzas-covarianzas.

\begin{tabular}{|c|c|c|c|c|c|c|c|c|c|}
\hline & RAC & $\mathrm{FFI}$ & SMC & $\mathrm{FOI}$ & CMR & GAC & CFO & MFC & MIC_D \\
\hline RAC & 0,63 & $-0,17$ & $-0,01$ & $-0,09$ & $-0,24$ & $-0,12$ & 0,05 & 0,63 & $-0,17$ \\
\hline $\mathrm{FFI}$ & $-0,17$ & 763,25 & 5,81 & $-7,79$ & 17,10 & $-4,08$ & $-9,05$ & $-0,17$ & 763,25 \\
\hline SMC & $-0,01$ & 5,81 & 49,00 & $-2,91$ & 4,10 & $-0,61$ & $-12,85$ & $-0,01$ & 5,81 \\
\hline FOI & $-0,09$ & $-7,79$ & $-2,91$ & 62,27 & $-11,19$ & 0,40 & 6,01 & $-0,09$ & $-7,79$ \\
\hline CMR & $-0,24$ & 17,10 & 4,10 & $-11,19$ & 100,76 & 2,14 & 0,30 & $-0,24$ & 17,10 \\
\hline GAC & 0,02 & $-1,00$ & $-1,06$ & $-0,17$ & $-0,82$ & $-0,18$ & 1,06 & 0,02 & $-1,00$ \\
\hline CFO & $-0,12$ & $-4,08$ & $-0,61$ & 0,40 & 2,14 & 5,05 & 2,37 & $-0,12$ & $-4,08$ \\
\hline MFC & $-0,01$ & 0,78 & $-2,37$ & 0,92 & 6,61 & $-1,88$ & 4,88 & $-0,01$ & 0,78 \\
\hline MIC_D & 0,05 & $-9,05$ & $-12,85$ & 6,01 & 0,30 & 2,37 & 83,27 & 0,05 & $-9,05$ \\
\hline
\end{tabular}

Con los 9 fondos se construyen los portafolios eficientes aplicando la teoría de Markowitz, para tal efecto se utiliza programación no lineal en Solver, que permite maximizar el rendimiento a cierto nivel de riesgo (volatilidad) a través de la matriz de varianzas-covarianza y el promedio de los fondos (ver tabla 4).

Por su parte, se observó que en el caso de los fondos MIC_B y MIC_D existe una perfecta correlación, en tal sentido MIC_B no ingresó al portafolio (se discriminó).

A continuación, se analizó la participación de cada uno de los fondos en 63 portafolios eficientes con su respectiva rentabilidad y riesgo, simulados con Solver de Excel. El portafolio de mínima varianza generó un rendimiento de 1,878\% al menor riesgo posible de 0,578\%, discriminando la participación del fondo MIC_D. El portafolio cuenta con participación del fondo RAC en un 53,99\%, seguido de GAC 31,47\%, CFO 9,8\%, MFC 1,88\%, SMC 1,58\%, FOI 0,77\%, CMR $0,38 \%$ y finalmente $\mathrm{FFI}$ con $0,13 \%$

\section{Construcción del portafolio óptimo}

Para determinar el portafolio óptimo se tomó el máximo desempeño de los portafolios eficientes a través del índice de Sharpe $0,3 \%$, y la tasa libre de riesgo se consideró como una aproximación de los bonos del tesoro en un plazo de 8 años con un rendimiento del 3\%. Según se muestra en la tabla 5, el portafolio óptimo está compuesto por cuatro fondos cerrados y un abierto, con relación a la moneda se da preferencia a bolivianos y tienen una calificación de riesgo entre $A A 1-A 2$, el resultado final se detalla en el orden de participación en la siguiente tabla. 
Tabla 5. Portafolio óptimo de fondos de inversión.

\begin{tabular}{llcccccc}
\hline Serie & \multicolumn{1}{c}{ Fondos de Inversión } & Participación & $\begin{array}{l}\text { Cal. de } \\
\text { Riesgo }\end{array}$ & Tipo & Moneda & SAFI \\
\hline MFC & $\begin{array}{l}\text { Fondo de Microfinancieras } \\
\text { Fondo de Inversión Cerrado } \\
\text { (MICROFIC) }\end{array}$ & 53,9 & A2 & Cerrado & BOB & $\begin{array}{l}\text { BISA SAFI } \\
\text { S.A. }\end{array}$ \\
SMC & $\begin{array}{l}\text { Sembrar Micro Capital } \\
\text { Fondo de Inversión Cerrado }\end{array}$ & 25,3 & A2 & Cerrado & BOB & $\begin{array}{l}\text { Capital }+ \\
\text { SAFI S.A. }\end{array}$ \\
MIC_D & $\begin{array}{l}\text { Microfinanzas - Fondo de } \\
\text { Inversión Cerrado Serie D }\end{array}$ & 17,6 & AA1 & Cerrado & BOB & $\begin{array}{l}\text { Fortaleza } \\
\text { SAFI S.A. }\end{array}$ \\
FFI & $\begin{array}{l}\text { Fondo de Inversión Cerrado } \\
\text { Fortaleza Factoring } \\
\text { Internacional }\end{array}$ & 2,8 & AA3 & Cerrado & USD & $\begin{array}{l}\text { Fortaleza } \\
\text { SAFI S.A. }\end{array}$ \\
FOI & $\begin{array}{l}\text { Fortaleza Interés + Fondo de } \\
\text { Inversión Abierto Corto } \\
\text { Plazo }\end{array}$ & 0,4 & AA2 & Abierto & BOB & Fortaleza \\
SAFI S.A.
\end{tabular}

Se determina que para el periodo estudiado el portafolio óptimo genera un rendimiento de $4,1 \%$ y el riesgo es de 3,65\%, con relación a la diversificación el orden de participación es MFC 53,9\%; SMC 25,3\%; MIC_D 17,6\%; FFI 2,8\% y FOI 0,4\%.

\section{CONCLUSIONES}

$\mathbf{S}$ e concluye que es posible optimizar el rendimiento de inversión en SAFls utilizando el Modelo de Markowitz, puesto que su aplicación permitió determinar un portafolio óptimo para un periodo de tiempo dado, asimismo permite encontrar el portafolio eficiente y de mínima varianza (menor riesgo). Se valora el aporte práctico para la toma de decisiones de inversión en Bolivia, ya que los resultados permiten deducir que es factible aplicar el mismo tratamiento estadístico para otros periodos de tiempo, permitiendo construir portafolios de inversión que optimicen el rendimiento de los instrumentos y minimicen el riesgo diversificado en valores emitidos en la bolsa boliviana de valores.

En este estudio en particular se partió por determinar un portafolio eficiente a través de programación no lineal en Solver de Excel y análisis de correlación lineal de Pearson, cuyos 9 fondos no presentaban correlación entre sí. Posteriormente se determinó que el portafolio de mínima varianza está compuesto por 8 fondos y genera un rendimiento de 1,878\% al menor riesgo posible de $0,578 \%$. Por su parte, el portafolio óptimo está compuesto por 5 fondos y genera un rendimiento de 4,1\% y el riesgo es de 3,65. Asimismo, se determinó que para el portafolio de mínima varianza el fondo RAC participa en un 53,99\% seguido de GAC 31,47\%, CFO 9,8\% y los 
demás 4,74\%, con relación a la diversificación en el portafolio óptimo, el orden de participación es MFC 53,9\%, SMC 25,3\%, MIC_D 17,6\%, FFI 2,8\% y FOI 0,4\%.

\section{REFERENCIAS}

ASFI (2014). Manual de cuentas para entidades financieras. Sociedad Administradora de Fondos de inversión. Bolivia

ASFI (2015). Fondos de Inversión. Sociedad Administradora de Fondos de inversión. Bolivia

Asociación de Bancos Privados de Bolivia (2013). La banca en cifras, [en línea]. La Paz: ASOBAN. Disponible en: http://www.asoban.bo [2014, 5 de marzo]

Banco Central de Bolivia (2014). Informe de Política Monetaria, [en línea]. La Paz: Asesoría de Política Económica. Disponible en: http://www.bcb.gob.bo [2014, 10 de mayo]

Castaño Celestino (2008). Valoración de pequeñas empresas: una aplicación a la marca "denominación de origen dehesa de extremadura". Departamento de Economía de la Empresa y Contabilidad. Facultad de Ciencias

Fabozzi, F. J., Markowitz, H. M., \& Gupta, F. (2008). Portfolio selection. Handbook of Finance, 2

Lawrence Gitman, Chad Zutter (2012). Principios de Administración Financiera (12va Ed.). (Trad. A.
Enriquez). México: Pearson. (Original en inglés 2011)

Markowitz Harry (1952). Portfolio Selection. The Journal of Finance, Vol. 7, No. 1. p. 77-91

Modigliani, F., \& Miller, M. H. (1958). The cost of capital, corporation finance and the theory of investment. The American economic review, 48(3), 261-297

Ross Stephen, Westerfield Randolph, Jaffe Jeffrey (2009). Finanzas Corporativas (8va Ed.). (Trad. J. Gómez y P. Carril). México: McGraw Hill. (Original en inglés 2008)

Sharpe William (1964). Capital Asset Prices: A Theory of Market Equilibrium under Conditions of Risk. The Journal of Finance, Vol. 19, No. 3, p. 425442

Villarreal Jesús (2008). Administración financiera II, [en línea]. EUMED. Disponible en: http://www.eumed.net [2014, 13 de septiembre] 